\title{
Developing astronomy in Cuba
}

\author{
Ramón E. Rodríguez Taboada ${ }^{1}$ \\ ${ }^{1}$ Department of Astronomy, Institute of Geophysics and Astronomy, \\ 212 St. No. 2906, La Lisa, C. Habana 11600, Cuba \\ email: ramone@infomed.sld.cu
}

\begin{abstract}
The development process of astronomy in Cuba is analysed from the point of view of a person actually working in astrophysics. It is concluded that the key word for astronomy development is "engagement" between a mature partner and the new developing group. It can not be forgotten that only astronomers do astronomy and that should be the main goal: to develop people working in astronomy.
\end{abstract}

Keywords. Sociology of astronomy, astronomy in Cuba

\section{Introduction}

This talk is devoted to describing the present Cuban situation in astronomy development, from the viewpoint of a person actually working in astrophysics. To make easier to understand the situation, I will begin with a brief historical introduction. Later, the up-to-date situation is presented and the topics relevant to astronomy development are analyzed.

Arising from national needs, astronomical calculations are the only "native-born" branch of astronomy in Cuba. Sun and Moon rise and set, culmination and elongation times of Polaris, local circumstances of eclipses, and other relevant data for the public and economic activities were developed early in the beginning of the 20th century with the foundation of the National Observatory. The National Observatory conducted the calculation of tides and other services too.

With the space age advent and the space race between Russia and USA developing, new needs arose. Located in the western hemisphere, Cuba was an observational platform capable to provide the Soviet Union with the 24 hours solar patrol needed by its Space Agency System to protect men in orbit. This was the beginning of a very fruitful development of solar research in Cuba. Russia installed the instruments, trained the people to operate them, and gave the academic environment needed to develop the scientific work in solar physics, space weather, and related topics. Considering this experience, I will comment on astronomy development in Cuba.

\section{Historical review}

\subsection{Solar research development in Cuba}

First was the task we were given. We were faced with a very clear task to perform: namely, a solar radio patrol for proton event diagnosis.

To perform such a task, several institutes of the Soviet Academy of Sciences decided to install radiometers in Cuba. The radiometers were to provide the data to perform the scientific and practical work, but they required a qualified crew to operate and maintain them. The solution to this problem led to the development of solar research in Cuba. 


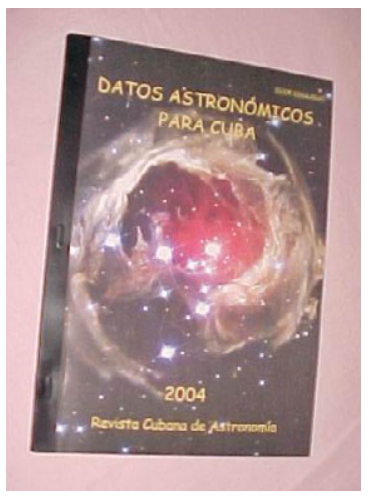

Figure 1. Astronomical Data for Cuba

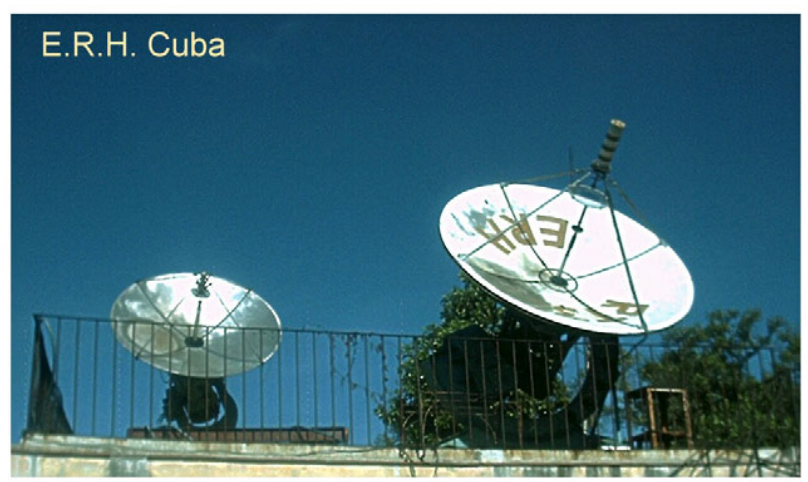

Figure 2. Havana Radio-astronomical Station, Cuba for SGD reports

In the beginning, the main task was to observe. Highly qualified teams of specialists from Soviet institutes visited the Institute of Astronomy in Cuba, to install equipment and to prepare the people to be in charge in Cuba. So we were qualified to operate and analyze the observations, and to calibrate and maintain the equipment and control their accuracy.

Very soon it was clear that people of the Havana Radio-astronomical Station could provide some kind of collaboration in solar research, and Soviet researchers leading projects involving the Havana Radio-astronomical Station observations included them in their work. For me, this was the turning point in the Cuban development of solar research. The Soviet groups investigating some edge problems in solar physics included Cuban specialists in their research projects, and received them for six-month periods in their institutes to provide the academic environment allowing to raise their qualification and later to prepare doctoral theses in accordance with their experience.

\subsection{Non-solar astronomy research}

The Cuban astro-climate is not good to develop an observational base. For this reason we have only participated in sporadic observations of planetary objects, as in the Soviet project Fobos and during the observational campaign of Halley's comet. These works were mainly astrometrical. Stellar variability was considered as a stellar research line to develop, but it was not successful in the absence of a strong partner to support the activity. 


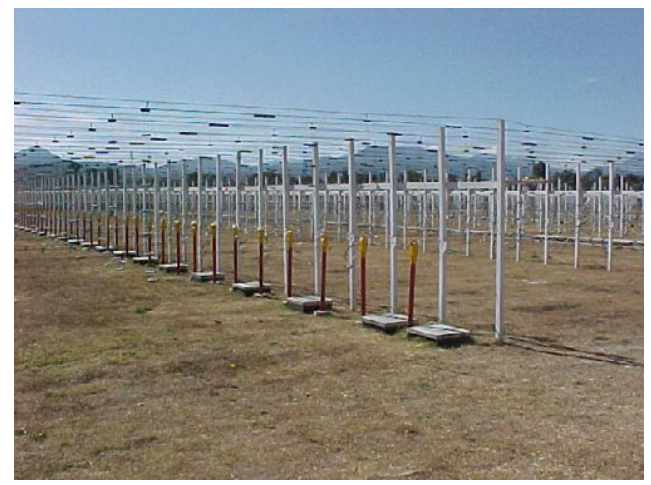

Figure 3. Scintillation telescope MEXART of IG-UNAM

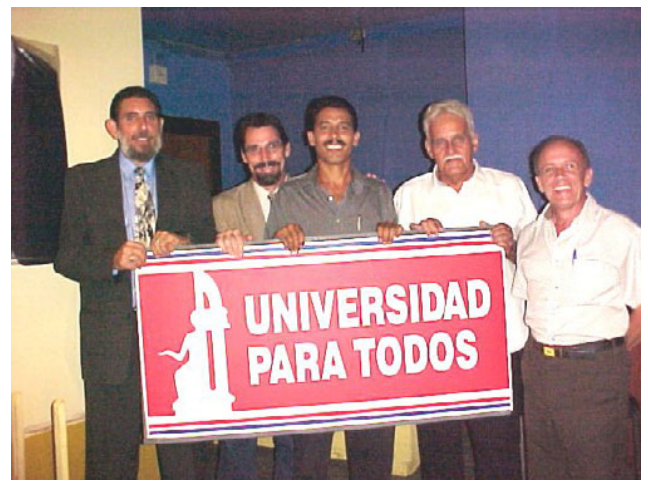

Figure 4. Staff for University for Every Body course of astronomy

\section{The present}

We are trying to develop stellar astronomy in collaboration with institutions capable to provide both the academic and technical environment. The IAC (Instituto Astrofsico de Canarias) received a Cuban researcher to prepare his doctoral thesis working within a project studying symbiotic systems, and Guanajuato University will receive another one to prepare her doctoral thesis in water masers in young stellar objects. But to continue developing stellar astronomy, we need to influence the public (an official) opinion to convince people they need groups working in astronomy.

How to do that? Publishing. Giving conferences talking about OUR work, not only like spectators of the science. Showing science is culture in modern times. Showing that projects in Astronomy can be cheap. This is very important! Astronomy is not a luxury for rich countries. The Cuban government is promoting a general increase of qualification and knowledge in the population. Cuba has a very complex educational system that includes TV lectures for elementary and secondary (junior and senior) levels in the formal structures of the national courses, but there are college level courses in the Old Adult University and not formal courses by TV programs too. Included in this effort there is a series of TV courses provided by a program named "Universidad para Todos" (University for Everybody). One of the courses passed by the Cuban TV during 2005 is "Elements of Astronomy". It consists of $30 \mathrm{TV}$ programs of one hour duration each, covering a wide spectrum of topics. The course is accompanied by a tabloid with the more important information of the lectures. The volume of the tabloid is of about 100 letter type pages including the figures. 


\subsection{Real possibilities}

I consider the Virtual Observatory concept the most appropriate in the near future, but to develop this possibility it is necessary to have an internet connectivity level that is not commonly provided in Cuba. Probably the more viable way to introduce this mode of astronomical research is adding people to the developing astronomical (solar and stellar) projects working in this way on collateral aspects of the main line. But any way, some kind of training is necessary and the need of a mature partner is evident. No matter, there is a lot of open and free software for astronomical use, the solar virtual observatory as it is in fact developing, is based on IDL, a system that is expensive.

Another research direction that we could develop is computational astronomy. But in this case we have not made contact yet with any specialist interested in accepting a Cuban researcher to work on solar system dynamics, galaxy interactions and related aspects.

\subsection{Contributions to astronomy development}

I have noted that in many cases researchers in Latin America look for partners ONLY in developed countries. We have some experience in South-South collaboration. Once Russia was not able to maintain the collaboration at the highest level, and requirements for solar patrol and research diminished. So we searched for common research interests in the area and found that the National Autonomous University of Mexico in its Institute of Geophysics was working in solar research with similar goals. After a few years of collaboration and academic exchanges supported by CONACyT grants, we have developed a strong contact with Mexican solar researchers and Cuban engineers are working in MEXART, a Mexican project including collaboration with India and Japan. Given that the formation of qualified specialists is one of the main aspects of astronomical development, we are working to include a post-graduate level course of Astrophysical Plasmas Basics that we give in the Faculty of Physics of the University of Havana as in the INAOE (Puebla, Mexico) doctoral courses.

\section{Concluding remarks}

From my experience "engagement" is the key word for astronomy development in developing countries. Efforts to develop astronomy in developing countries succeed only if an astronomer with experience in the field guides the firsts steps of those people that are initiating this work in the fields where they have experience. Medical doctors do medicine, engineers buildings, etc. If you give resources to doctors they will use it to develop medical assistance, if to engineers to build. Only astronomers can do astronomy, so the resources that you want to be dedicated to develop astronomy should be given to astronomical projects including astronomers and/or "astronomers to be" from the developing countries.

Astronomy can not be developed without an appropriate academic environment, and we do not have it at present. Some countries in Latin America have better conditions to collect together small groups of people working in astronomy, but their more mature researchers have not taken conscience of their importance and possible influence in the development of astronomy in our area. It is not "only" about financial resources, it is also about "real collaboration" with mature partners and common research goals. From my point of view, only by working, and making astronomical research, will astronomy be developed. Science is just one, and it seems to me improper to waste any human resources available. 